\title{
ACHEGAS AO DICIONĀRIO DOS ANIMAIS DO BRASIL, DE RODOLPHO VON IHERING
}

\author{
João de Paiva Carvalho $\left({ }^{1}\right)$ \\ e Paulo Sawaya $\left({ }^{2}\right)$ \\ $-\mathrm{B}-$
}

BACÁCA - Nome que, em Manaus, se dá ao Anambé-azul, Cotinga cayana (L.). Ave da fam. Cotingidae.

BABAQUEIRO - Espécie de Cação, conhecido por êsse nome no litoral do Estado de São Paulo.

BACALHAU - Nome dado ao Mangangá liso, Nautopaedium porosissimum (vaL.), muito comum em águas brasileiras. Peixe da fam. Batrachoididae.

BACURÁU BRANCO - Ave Caprimúlgida, do norte do Brasil, Chordeiles rupestris rupestris (SPIX). Fam. Nyctibiidae.

BAGRE DE ÁGUA DOCE - Denominação vulgar da espécie de peixe Luciopimelodus platanus GÜNTHER.

BAGRE DE LAGÔA - É a espécie ictiológica conhecida cientìficamente por Rhamdia sebae cuv. \& VAL.

BALEOTE - Nome que se dá ao filhote de Baleia (Cetáceo).

BANDEIRINHA - Nome dado a borboletas diurnas da fam. Lycasidae, Techla schion L. e T. regalis CHAM., O mesmo que Piraíba.

BARATINHA DA PRAIA - Crustáceo Isopode muito comum nas praias. Não vivem pròpriamente no mar, mas acompanham a linha da maré. Comem detritos; respiram por meio de brânquias localisadas no abdomen. Apresentam o interessante fenômeno da mudança da côr e do ritmo noturno. No litoral brasileiro ocorre comumente a espécie Ligia exótica (ROUx).

(1) Do Instituto Oceanográfico, da Universidade de São Paulo.

(2) Do Departamento de Fisiologia Geral e Animal. - Fae. Fil. Cien. Letras, da Univ. de São Paulo, Caixa Postal, 2926. São Paulo. 
BARIZOS - Denominação vulgar dada pelos índios Napos ao Macaco de cheiro.

BARONA - Espécie de Manjuba ocorrente na bacia fluvial do Parnaíba, E. do Piauí, pertencente, provàvelmente, ao gênero Anchoviella.

BARRACA - Caranguejos do gênero Stenorhynchus.

BARRIGUDO - Símios da Amazônia, do gên. Lagothrix. São macacos obesos, de cêrea de $60 \mathrm{cms}$ de altura, de pêlo curto, lanudo. Conhecem-se várias espécies: Lagothrix lagothrica peruensis LöNNB., vulgarmente conhecido como Barrigudo Comum; $L$. lagothrica lagothrica (нUмв.), chamado Barrigudo Cinzento. É cinzento-amarelado ou avermelhado, com a cabeça e as extremidades quase pretas, sendo de todos os macacos do Brasil um dos que mais se presta à domesticação, afeiçoando-se às pessoas com que convive, sem o perigo dos outros macacos que mordem quando irritados ou por ocasião do cio; L. infumata (SPIX) é mais escuro e conhecido por Barrigudo Pardo, do Alto Amazonas. Todos êstes macacos domesticam-se, mas quando na mata mostram-se atrevidos e irritados. Vide "Caridagueres" e "Caparu". A. Miranda afirma, ao contrário de outros escritores, que os barrigudos sempre são de índole mansa.

BARROSO - Espécie de Cação, Gynglimostoma cirratum (BONNATERre), também conhecido por Lixa de lei.

BASILISCO - Grande lagarto verde e pardo - Basiliscus americanus - barrado de negro, com a região cefálica, o dorso e a cauda providos de crista, encontrado desde o sul do México até o norte da Amazônia.

BATUIRINHA - Ave perteneente às pequenas espécies de Batuira, Tringa solitaria solitaria wiLson e Actitis macularia (L.).

BEIJA-FLOR D'ÁGUA - Ave Piciforme, da fam. Galbulidae, ocorrente em quase todo o Brasil, Galbula rufoviridis rufoviridis CABANIS.

BEIJA-FLOR DE BICO VERMELHO - Fam. Trochilidae, da região meridional do Brasil, Chlorostilbon aureoventris berlepschi oLIVEIRA PINTO.

BEIJA-FLOR DE PENACHO - Da região sudeste do Brasil, Stephanoxia lalandi (vIEILLOT).

BEIJA-FLOR DO MATO - Espécie do sudeste do Brasil, Rhamphodon naevius (DUMONT), conhecido, no Ceará, por Beija-flor pardo.

BEIJA-FLOR DO RABO BRANCO - Do centro e do leste do Brasil, Anisoterus pretrei (DELATTRE \& LESSON).

BEIJA-FLOR GRANDE - Nome atribuído à espécie Eupetomena macroura simoni hellmayr, do noroeste brasileiro. Nome que se 
dá, também, às galbúlidas Galbula rufoviridis rufovridis CABANIs e G. trombacea ruficauda CUVIER.

BEIJA-FLOR PRETO - Espécie da região leste do Brasil, Melanotrochilus fuscus (VIEILLOT).

BEIJA-FLOR VERMELHO - Espécie da região leste e norte da América Meridional, Chrysolampsis alatus (L.).

BEM-TE-VI CAVALHEIRO - Ave tirânida, Myiodynastes maculatus maculatus (MÜLLER), da Amazônia.

BEM-TE-VI DO MATO - Ave comum em quase todo o Brasil, Myiodinastes solitarius ( VIEILLOT).

BERóE - Ctenóforos cosmopolitas - Beroe ovata -, dotados de corpo gelatinoso e transparente, de forma cônica, provido de numerosos cílios; bôca e farínge muito ampla, dotadas de canais gastrovasculares na zona meridional, com numerosas ramificações. Essa belíssima campânula transparente é comum em quase tôdas as baías e enseadas, onde aparecem em determinadas épocas do ano. São animais muito interessantes. Na estrutura do corpo mais de $90 \%$ correspondem à água.

BESUGO - Nome, provàvelmente de origem lusitana, que designa 0 Pargo. Denominação que se dá, também, em certos lugares, ao Panaguaiú.

BICHA - Diz-se, freqüentemente, de todos os vérmes e répteis. Nome que se dá à Onça.

BICHO CABELUDO - Nome atribuído a várias lagartas de borboletas.

BICHO DE CHARUTO - Nome que, no Rio Grande do Sul, designa a larva polífaga do inseto Oiketicus geyeri (BERG.,).

BICHO DE CHIFRE - Nome de várias lagartas de borboletas.

BICHO DE RUMO - Nome de uma borboleta Papilionidae, Papilio anchisiades capys HUBN.,

BICHO DO CACAU - Nome da larva de uma borboleta noturna da fam. Phycitidae, Myelois duplipunctella RAG.,

BICHO DO CAFÉ - Nome da larva de uma borboleta noturna da fam. Lyonetiidae, Leucoptera cofeolla GUER.,

BICHO DO MINEIRO - Designação da larva de uma borboleta noturna da fam. Lyonetiidae, Leucoptera cofeolla GUER., O mesmo que Bicho do Café.

BICO DE FERRO - Ave da fam. Fringillidae, Saltator similis similis LAFRESNAYE \& D'ORBIGNY. 
BICO DE LACRE - Ave Passeriforme africana - Estrilda cinerea (VIEILLOT), introduzida no Brasil e perfeitamente adaptada ao nosso clima, sobretudo nos Estados do Rio de Janeiro e S. Paulo onde, de há muito, é encontrada em estado de absoluta liberdade.

BICO MIÚDO - Pássaro da fam. Rostratulidae, das fronteiras do Brasil, Uruguai, Paraguai e Argentina, Nycticryphes semi-collaris (VIEILLOT).

BICO DE PRATA - Nome de certos Tiés dos gêneros Ramphocelus e Pipra. Ave Passeriforme.

BICO DE VELUDO - Tanagrida do Brasil meridional - Schistochlamys ruficapillus ruficapillus ( угегLот). Ave Passeriforme.

BICO VERMELHO - Nome dado a um Papacapim da região este-meridional do Brasil, Sporophila leucoptera cinereola (TEMMINCK). Ave Passeriforme.

BICUDA - Serpente Colúbrida da série aglifodonte, da subfamília Colubrinae, Uromacerina ricardinii (PERACA), até agora só encontrada em São Paulo. Dá-se êsse nome a um exemplar da subfamília Boiginae, da série opistoglifodonte, Rhinostoma guianense (TROSCHELL) própria da zona sub-equatorial e a um representante do gênero Oxybelis, $O$. acuminatus (WIED), das zonas equatorial e tropical.

BICUDO PRETO - Nome que, na Amazônia, se dá ao Bicudo, Cryzoborus crassirostris maximiliani CABANIS. Ave Passeriforme.

BIRÍ-BIRÍ - Peixe fluvial da fam. Characinidae, Leporinus nigrotaeniatus KER.

BIRÚ-LISTRADA - No norte do Brasil, nome da Cobra-cipó, Eudryas boddaertii (SENTZEN), restrita às regiões setentrional e ocidental do Brasil, indo, porém, até o México.

BLÁTIDAS - Ordem de Insétos a que pertencem as Baratas.

BIZOGUE - Macaco do Amazonas, Callicebus torquatus purinus thomas. Nome que se dá, também, à espécie Callicebus baptista LöNNBERG.

BÔBO - Nome dado a uma ave Procelariforme do R. G. do Sul, Puffinus puffinus puffinus (BRÜNNICH), ocorrente na costa meridional do Brasil.

BôCA DE COBRA - Nome que, em Iguape (E. de S. Paulo), designa a Sardinha, Lycengraulis batesii (GÜNTHER).

BÔCA DE SAPO - Em Mato Grosso, designação de Jararaca do rabo branco, Bothrops neuwiedii fluminensis aMARaL, espécie que freqüenta também o E. de Minas Gerais e o norte de S. Paulo. 
BÔCA LISA - Bagre fluvial, Tachysurus upsulonophorus (EIGENMANN \& EigenmanN), também conhecido por Papai ou Bagre Papai.

BÔCA NEGRA - Peixe marinho, do Nordeste, da fam. Lutjanidae, gênero Neomaenis.

BOIA - Nome de uma borboleta diurna, da fam. Morphoidae, Morpho hercules DALM.

BOICHUMBEGUACुU - Nome da Cobra coral, Micrurus lemniscatus (L.), da fam. Elapidae. Mato Grosso. Vide Cobra coral e Ibiboca.

BOI DE CARRO - Peixe Actinopterygii, da fam. Loricariidae, conhecido por êsse nome no rio Parnaíba, E. do Piaú, Loricaria typus BLEEKER.

BOIOBU - Usa-se êsse nome, na região central e meridional do Brasil, para designar uma serpente Colúbrida da fam. Boiginae, Philodryas aestivus (DUMÉRIL \& BIBRON), também conhecida por Cobra verde.

BOIPEMI - Serpente Colúbrida da subfam. Boiginae, Tomodon dorsatus DUMÉriL \& BIBRoN, conhecida por êsse nome no Brasil Central. Veja-se também Corre-campo.

BOIPINIMA - Na região meridional do Brasil, indica a Cobra coral - Micrurus frontalis (DUMÉRIL \& BIBRON). Vide Cobra coral.

BOIPIRANGA - Nome que, no sul e oeste do Brasil designa a Cobra coral, Elapomorphus tricolor DUMÉRIL \& BIBRON. Vide Cobra coral falsa.

BOIUBU - Nome atribuído a duas cobras do gênero Philodryas, $P$. aestivus (DUMÉRIL \& BIBRON) e P. viridissimus (L.).

BOMBUDAS - Denominação local, da região de Iguape e Cananéia, aplicada a uma espécie de Manjuba. Trata-se do Engraulida Anchoviella hubbsi HILDEBRAND, de grande importância econômica para as populações que habitam o rio Ribeira de Iguape.

BORBOLETA CORUJA - Nome dado a uma borboleta da fam. Brassolidae, Caligo eurilochus brasiliensis.

BORBOLETA CORUJINHA - Nome dado a várias borboletas da fam. Brassolidae, do gênero Opsiphanes.

BORBOLETA ESPELHO - Nome de várias borboletas da fam. Saturnidae, do gênero Rothschildia.

BORBOLETA RUBÍ - Borboleta da fam. Nymphalidae, Anaea rhyphea HUBN.,

BOTÃO DE OURO - Sapinho diminuto, habitante de sólos húmidos e bromeliáceas, Brachicephalus ephipium, comum no Brasil. 
BRANQUINHO - Sob êste nome, quase sempre pronunciado no feminino, designa-se, na Amazônia, a espécie Anodus zetior SPIx. Pela mesma denominação é conhecido um representante da subfam. Curimatinae, Acuticurimata macrops (EIGENMANN \& EIGENMANN), do Ceará

BRAQUICEROS - Divisão dos Diptera, composta de insétos portadores de antênas curtas.

BRAQUIURO - Divisão dos crustáceos decápodos providos de abdômen oculto sob o cefalotórax, tais como os Sirís e Caranguejos.

BRIBA - Espécie de Cobra de vidro, Diploglossus fasciatus GozLd, do extremo norte do Brasil.

BRIOZOÁRIOS - Classe de Tentaculados ou Moluscóides, de água dôce e salgada, bem representada no Brasil.

BROCA DO CAFE - A êste verbete acrescentaríamos : O nome científico adotado é Hypotenemus hampei (FERRARI). Experiências ùltimamente efetuadas sôbre a biologia desta praga dos cafèsais demonstraram a grande resistência da larva aos inseticidas. Tem-se estudado também o metabolismo desta larva com resultados bem interessantes.

BROMA - Diz-se das brocas em geral, que atacam as madeiras.

BUGIU RUIVO - Espécie de Bugiu muito característico - Alouatta fusca, do Amazonas, Bahia, Espírito Santo e Minas Gerais.

BÚSSOLA - Nome vulgar de uma borboleta noturna, da fam. Ceratocampidae, Neocarnegia basirei SAHs.,

Sinônimo

Baca-aí

Bacurau preto

Bacurau de bando

Badejo pintado

Bagre branco

Bagre gurí

Bagre mole

Bairú

Balaieiro

Banana

Bandeirado

Bandolim

Barreiro

Bacorá

Badejo ferro

Badejo mira

$$
\text { Verbete }
$$
Saracura
Anambé branco
Bacurau branco
Garoupa pintada
Bagre urutú
Tamboatá
Cambéva
Mussurana
Jurupiranga
Maria Luiza
Bagre mandim
Albacóra
João de Barro
Boicorá
Badejo
Badejete 


$$
\text { Sinônimo }
$$

Baiacú-ára

Baiacú de espinho

Bagre fita

Bandeirado

Bendim

Bariguí

Bastião

Batuirão

Batuqueiro

Beatriz

Beija-moça

Bejaquí

Bem-te-vi de cabeça de estaca

Bem-te-vi carrapateiro

Bem-te-vi do bico largo

Bem-te-vi de cerda

Bem-te-vi do gado

Bem-te-vi escuro

Bem-te-vi preto

Bem-te-vi rajado

Bernardo eremita

Bicho de conta

Bicho de porco

Bico de agulha

Barata noiva

Bico de fogo

Bico de furo

Bico de pato

Bico preto

Bico dôce

Bico revôlto

Bicudo do norte

Bigode

Bigodinho

Bôca de fogo

Bodinho

Boiaçú

Boi-cipó

Boicorá

Boiçá

Boiçununga

Boi-marinho

Boiobú

Bico de cravo

Boiquíra

Boirú
Verbete

Baiacú-pinima

Baiacú. Baiacúguaima

Bagre bandeira

Bandeira. Bagre bandeira

Bagre mandim

Biriguí

Cricrió

Galinhóla

Trinca-ferro

Niquim da pedra

Parú da pedra

Baiacú

Suirirí

Suirirí

Bem-te-vi do bico chato

Bem-te-vi

Carrapateiro

Bem-te-vi cavaleiro

Bem-te-vi do mato. Siriritinga

Bem-te-vi cavaleiro

Paguro

Baratinha

Bicho de pé

Beija-flor d'água

Barata deseaseada

Bico de braza

Avinhado. Curió

Jurupóca

Bicudo

Focinhudo e Teiú

Massaricão

Bicudo. Bicudo preto

Coleirinha

Coleirinha

Coreoróca

Sebastião

Sucurí

Cutimbóia

Boicoral

Sucurí

Boicininga

Peixe-boi

Boiubí

Bico de braza. Tangurupará

Cascavel

Mussurana 


$$
\text { Sinônimo }
$$

\section{Boitiabóia}

Boitiapóia

Bonito do campo

Borá-cavalo

Boraquíra

Bôto vermelho

Bracajá

Brijára

Broca do figo

Brujajára

Bugiu labareda

Busano

Buréva

Buvuarí

Buzo

$$
\text { Verbete }
$$

Cobra cipó

Sucurí

Terê

Boraboi

Codorna

Pirajaguára

Tracajá

Brujarára

Broca

Brujarára

Guariba vermelho

Guzano

Bagre d’água dôce

Acará bandeira

Buzio 